\title{
Impulsive Increase of Galactic Cosmic Ray Flux Observed by IceTop
}

The IceCube Collaboration ${ }^{\dagger}$, Pierre-Simon Mangeard ${ }^{\ddagger}$, Pradiphat Muangha ${ }^{\sharp}$, Roger Pyle $^{\natural}$, David Ruffolo $\sharp$, and Alejandro Sáiz $\sharp$

†http://icecube.wisc.edu/collaboration/authors/icrc17_icecube

\$ University of Delaware, Newark, DE 19716, USA

\# Mahidol University, Bangkok 10400, Thailand

${ }^{\natural}$ Pyle Consulting Group, St. Charles, IL 60174, USA

E-mail: evensondudel.edu

On 2017 January 18 scaler rates in the IceTop detectors at the South Pole revealed an impulsive increase in the galactic cosmic ray flux lasting a few hours. In addition to the neutron monitor at Pole the event was detected clearly by the Mawson neutron monitor and faintly at Jang Bogo. No other neutron monitors appear to have seen the increase. The event was in many ways reminiscent of the 2015 June 22 event observed by the GRAPES muon detectors. Both events occurred during the declining phase of a Forbush decrease, at a time of increasing geomagnetic activity, and were observed by a limited number of neutron monitors with similar asymptotic directions. The magnitude of the impulse was in both cases such that the flux returned briefly to approximately the pre-decrease level. Distinctly unlike the 2015 June 22 event, a changing geomagnetic cutoff cannot explain the 2017 January 18 event because the cutoff at South Pole is nearly zero and the detector response is atmosphere limited. We therefore interpret the 2017 January 18 event in terms of the structure of the Forbush decrease and (possibly changing) asymptotic directions. With our interpretation of the January event in mind we also comment on possible alternative interpretations of the GRAPES event.

Corresponding author: Paul Evenson*

University of Delaware, Newark, DE 19716, USA

35th International Cosmic Ray Conference

10-20 July, 2017

Bexco, Busan, Korea

* Speaker. 


\section{Introduction}

IceCube is a cubic-kilometer scale neutrino detector completed in 2010 installed at the geographic South Pole [ []]. Reconstruction of the direction, energy and flavor of the neutrinos relies on the optical detection of Cherenkov radiation emitted by charged particles produced in the interactions of neutrinos in the surrounding ice or the nearby bedrock. The Cherenkov light is measured by Digital Optical Modules (DOMs) containing photomultipliers deployed in the ice between depths of $1450 \mathrm{~m}$ and $2450 \mathrm{~m}$. IceTop is an air shower array with 81 stations located above the in ice installation. A station consists of two Cherenkov "tanks" which are $2000 \mathrm{~kg}$ blocks of clear ice using standard DOMs to measure Cherenkov radiation emitted by secondary charged particles. One DOM in each tank is operated at high gain and the other at low gain in order to extend the dynamic range of the tank.

To measure $\mathrm{GeV}$ cosmic ray fluxes we use count rates from two discriminators in each high gain DOM, termed SPE (Single Photo Electron) and MPE (Multi Photo Electron). The SPE are set at selected thresholds ranging between 1 and 20 photoelectrons while the MPE are all set near 20 photoelectrons. Setting the discriminator level on a DOM "tunes" the response function of the DOM. The array of different thresholds allows determination of the energy spectrum and time structure of variations in the interplanetary cosmic ray flux. See [䧃] for a more extensive discussion of the energy response of IceTop. There is also a 3NM64 neutron monitor [] $]$ at the South Pole with response peaking at lower energy than any of the IceTop thresholds. Lead free neutron detectors with yet lower energy response that we refer to as the "Polar Bares" (although a more common name is "Moderated Neutron Detectors") [Q] are installed in the main station. For details of the neutron detectors at the South Pole see [四].
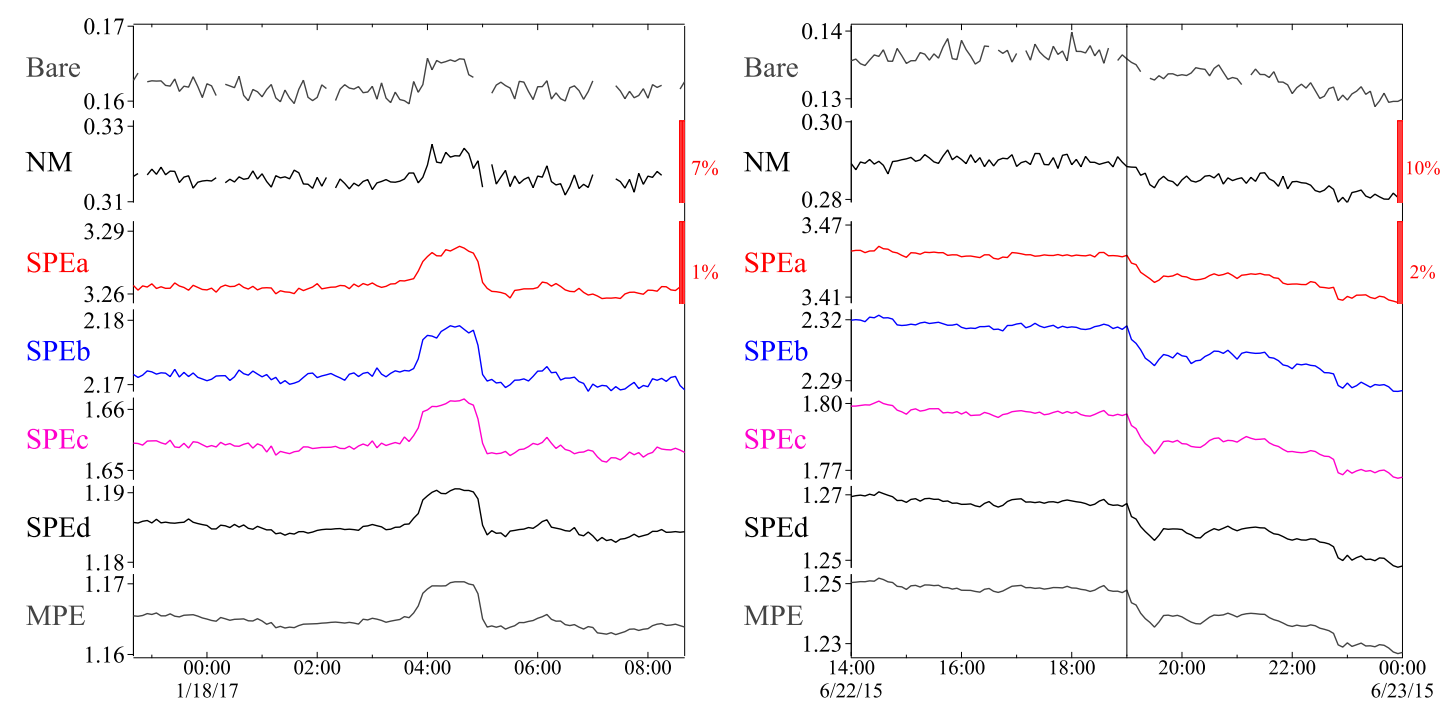

Figure 1: Left: Impulsive increase in cosmic ray flux seen by IceTop and the South Pole neutron detectors on 2017 January 18. Right: IceTop response at the time of the impulsive increase reported by GRAPES on 2015 June 22. All plots are logarithmic with rates expressed in $\mathrm{kHz}$.

On 2017 January 18 IceTop observed the impulsive increase in the cosmic ray flux illustrated

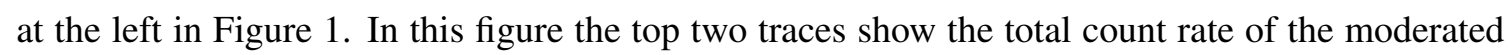


neutron detectors and 3NM64 neutron monitor respectively, while the next five traces give the average counting rate of IceTop DOMs grouped in order of increasing threshold. The similar-sized increase at all thresholds in IceTop and neutron detectors indicate that the impulsive increase has a hard spectrum, very close to that of the galactic cosmic rays. A small enhancement was also seen at the nearby neutron monitors at Mawson and Jang Bogo, but no others. Thus it is evidently a highly anisotropic fluctuation in the galactic cosmic rays - as opposed to a Ground Level Enhancement (GLE) resulting from solar energetic particles (SEP).

The event seen by IceTop was in many ways reminiscent of the 2015 June 22 pulse event observed by the GRAPES-3 tracking muon telescope in Ooty, India [ $\square]$. Both the IceTop and GRAPES events occurred during the declining phase of a Forbush decrease, at a time of increasing geomagnetic activity, and were observed by a limited number of neutron monitors with at similar geographic locations. The magnitude of the impulse was in both cases such that the flux returned briefly to approximately the pre-decrease level. An explanation proposed for the GRAPES event $[\square]$ is a temporary reduction in the geomagnetic cutoff amounting to $0.5 \mathrm{GV}$ to $0.7 \mathrm{GV}$ depending on the incident direction of the particles.

On the right in Figure $\mathbb{W}$ we show data from the South Pole detectors at the time the pulse event was observed by GRAPES. Since any SEP event gives a much stronger increase at low cutoff rigidity the absence of an increase at South Pole definitively confirms that the event is not a classic GLE. The clear depression in count rates at the South Pole at the time of the start of the GRAPES pulse (vertical line) was presumably produced by the same magnetic disturbance. Since the cutoff is zero at the South Pole, and therefore cannot decrease, the lack of a pulse in and of itself does not contradict the explanation proposed [ [ $]$ ] for the GRAPES event.

However, by the same logic, a changing geomagnetic cutoff cannot explain an increase such as seen on 2017 January 18 . The only explanation for the 2017 January 18 event is a significant anisotropy in the interplanetary cosmic ray flux. Whether the anisotropy was transient in time or was a result of changing asymptotic directions due to the active geomagnetic conditions at that time cannot be decided. With this picture of the 2017 January 18 event in mind we propose that the GRAPES event also was a manifestation of interplanetary anisotropy rather than a cutoff variation. Primarily we argue that a cutoff variation would make predictions for the world network of neutron monitors that are not in accord with observations.

\section{Geomagnetic Cutoff Variations}

The Princess Sirindhorn Neutron Monitor (PSNM) on Doi Inthanon in Thailand is close to GRAPES, both geographically and in cutoff rigidity. At PSNM the vertical cutoff, in geomagnetic quiet conditions, is $\approx 16.7 \mathrm{GV}$, whereas the directionally averaged cutoff is $\approx 17.3 \mathrm{GV}$. The counting rate at PSNM at the time of the GRAPES event is shown at the top of the left panel in Figure $\square$. The timing and structure of the pulse at PSNM is similar to that of the GRAPES pulse. Using our calculated yield functions for PSNM [目] we also find that the magnitude of the increase at PSNM is quite consistent with a cutoff reduction of approximately 0.6 GV. As Figure $\square$ shows, all neutron monitors show the onset of a large Forbush decrease near this time. Because of the Forbush decrease the identification of a pulse is somewhat subjective but it is clear that only a other few neutron monitors show a pulse as well defined as that at PSNM. For the monitors where the pulse 

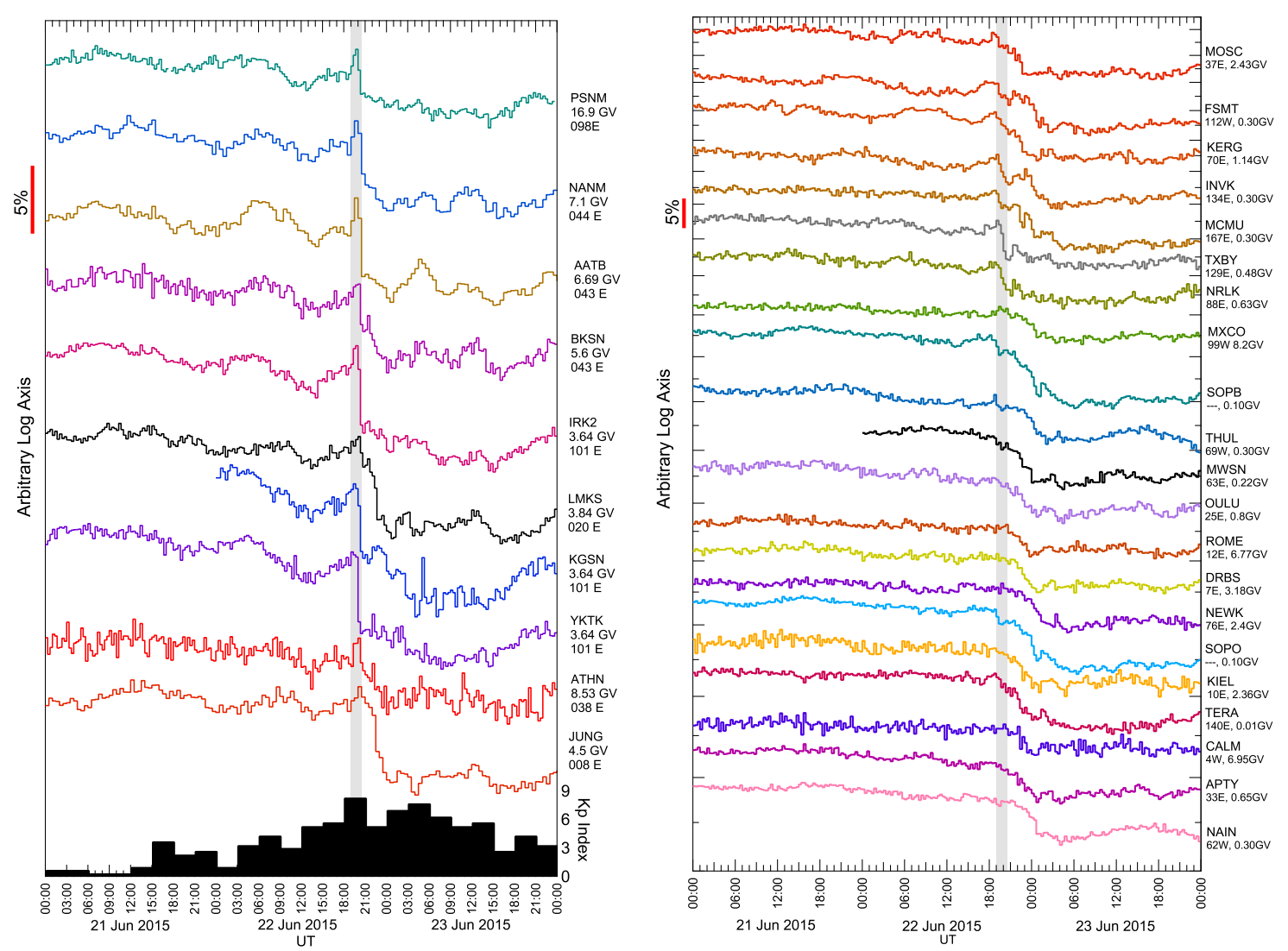

Figure 2: Left: Response of several neutron monitors having the largest increase at the same time as that observed by GRAPES on 2015 June 22. The Kp index of geomagnetic activity is shown in the lower panel. Right: Multiple neutron monitor observations ranked approximately by the intensity of the observed pulse. The approximate duration of the GRAPES pulse is indicated by the gray shading.

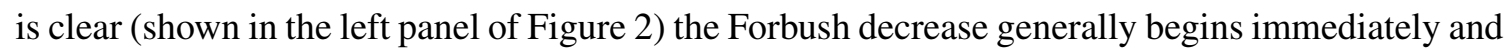
steeply following the pulse. For the other monitors the onset is not as sharp and is delayed by varying amounts. This behavior is characteristic of commonly observed Forbush decrease precursors. For a discussion and classification approach to such precursors, see[[]].

We also find that a cutoff variation localized to the vicinity of the GRAPES detector is unlikely. In the commonly used Tsyganenko [W] model of the response of the magnetosphere to perturbation by interplanetary disturbances any variation in the geomagnetic cutoff is a fully global process. In this model the geomagnetic field cannot be described as a simple superposition of the interplanetary field and the internal (IGRF) field. Rather, the disturbance of the solar wind perturbs global current systems, and it is these current systems that affect the cutoff. Since these current systems are global, any change in the cutoff must also be global. The left panel of Figure [ ] shows our calculation of cutoff changes predicted by the Tsyganenko model, performed by the method described in [\$]], as a function of $\mathrm{Kp}$, the geomagnetic parameter that is the input to the Tsyganenko model.

$\mathrm{Kp}$ is an index computed from the range of fluctuations in the horizontal component of the magnetic field measured at ground level at a specifically defined array of locations. For an excellent discussion of why the single parameter Kp provides a good description of the state of the 
magnetosphere see [미]. Note that it is an inescapable prediction of the Tsyganenko model that all locations on Earth have correlated changes in the geomagnetic cutoff, and indeed that cutoff changes are systematically larger for lower cutoffs.
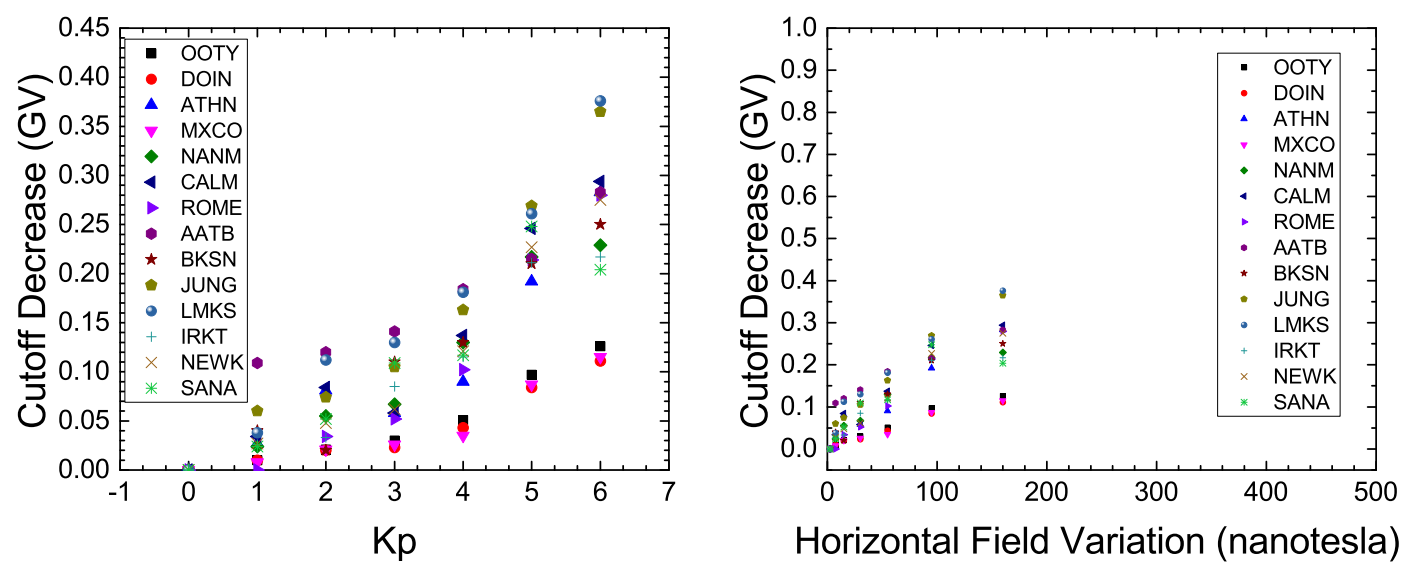

Figure 3: Cutoff reduction at various neutron monitors in the Tsyganenko model. Left: Plotted as a function of the single parameter in the model, integral values of the quasi logarithmic Kp. Right: Plotted as a function of the underlying linear magnetospheric parameter. The horizontal axis extends to $500 \mathrm{nT}$, which corresponds to $\mathrm{Kp}=8$, the approximate value needed for extrapolation in the 2015 June 18 event. The cluster of points are the same as those in the left panel and thus indicate the values of $\mathrm{Kp}$ on this scale.

Unfortunately for the present analysis the Tsyganenko model relies on a fit to data to derive coefficients that describe all of the current systems that are assumed to follow Kp. Data above $\mathrm{Kp}=6$ are too sparse to allow such a fit at these values. Consequently we cannot directly do the calculation for the $\mathrm{Kp}=8$ level at the time of the GRAPES pulse. However the model employs physically meaningful parameters that describe specific magnetospheric current systems so there is no reason to assume that the model itself fails at that time.

The right panel of Figure 3 shows the calculated cutoff decrease plotted as a function of the range of variation of the horizontal surface field, which is the underlying linear parameter of the quasi-logarithmic $\mathrm{Kp}$. The extent of the horizontal axis has been chosen to approximate the degree of extrapolation necessary to reach $\mathrm{Kp}=8$. Linear extrapolation of the cutoff change at Ooty and Doi Inthanon (the lowest sequence of points on the figure) yields a somewhat smaller value than the 0.6 GV that would produce the effect seen at these stations, particularly considering that Kp changes from 6 to 8 at that time, not from 0 to 8 . Exact agreement of the extrapolation with observation is not important to our argument. The key point is that the amount of extrapolation required is not so large that the model itself would fail in such a way as to modify the conclusion that cutoff changes are a global, not local, phenomenon.

\section{Geographic Distribution}

Figure $\mathbb{A}$ shows the distribution of neutron monitors at the time of the GRAPES event with symbols indicating our somewhat subjective classification of the appearance of a pulse at the monitor. Using the data in Figure $\square$ we have assigned (basically by eye) one of three categories to each 


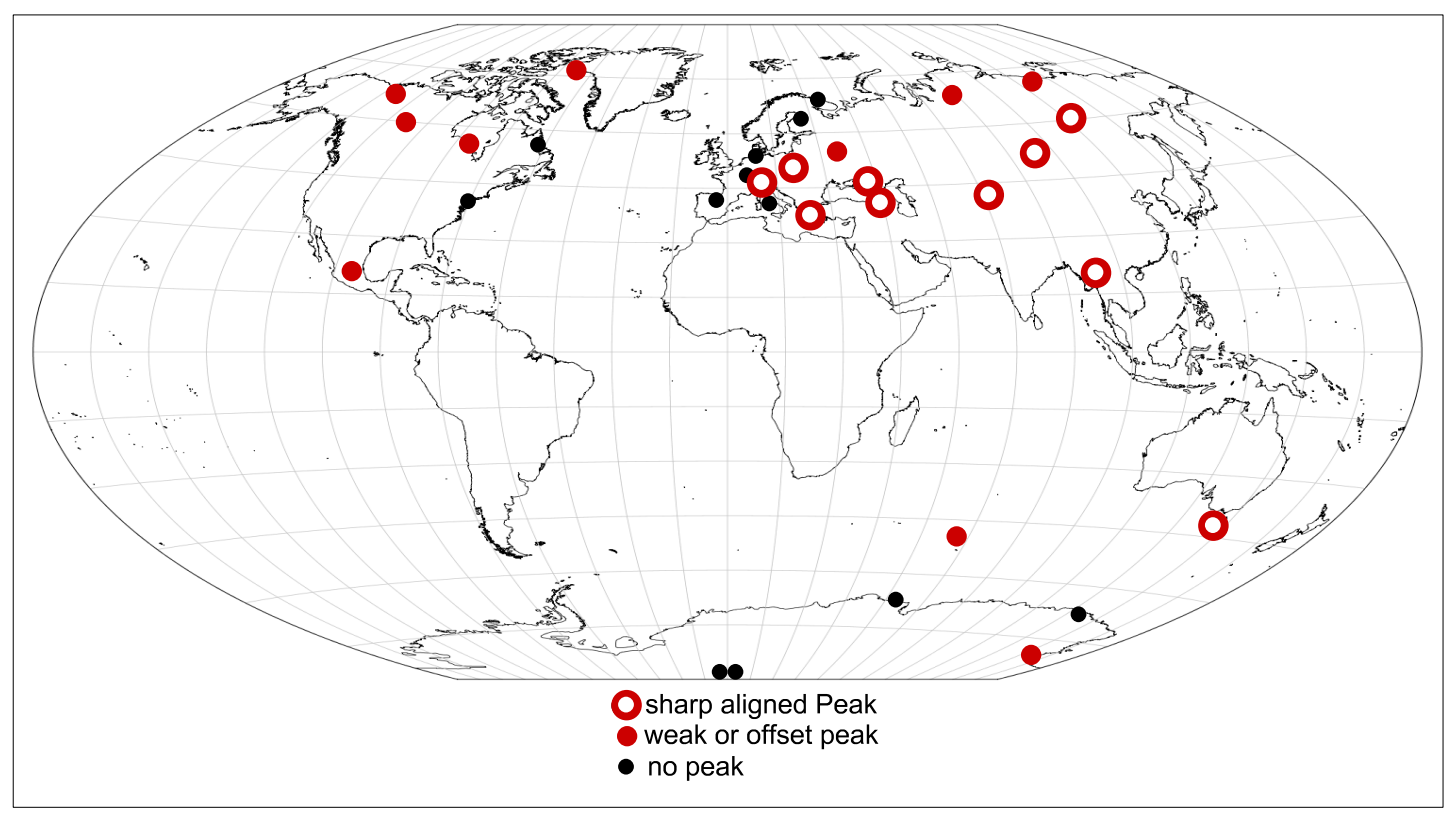

Figure 4: Geographic distribution of pulse visibility on 22 June 2015. Symbols indicate the location of neutron monitors classified as observing sharp aligned peaks, weak or offset peak, or no peak.

monitor: "sharp aligned peak", "weak or offset peak", or "no peak". The geographic distribution of these categories is well organized by location, as expected for an anisotropy effect. According to Figure 3$]$ changes in cutoff rigidity are not restricted to Asian longitudes. European stations have the strongest predicted cutoff changes yet most of them show no peak. This strongly argues against the interpretation of the GRAPES event in terms of a cutoff change.

\section{Discussion and Conclusions}

There is a general similarity in the morphology of the 2015 June 22 event observed by GRAPES and the 2017 January 18 event observed by IceTop. Both have a small, well defined but geographically localized increase in counting rate at the time of a magnetospheric disturbance. At the South Pole a lowering of the cutoff is impossible, so the only explanation for the 2017 January 18 event is anisotropy in the cosmic ray flux.

For the GRAPES peak on 2015 June 22 a cutoff change is possible, and the required magnitude of the cutoff change is consistent with an extrapolation of the Tsyganenko model. However the peak is not global as would be predicted by that model. The peak is also visible at several polar locations where the cutoff is so low that a change could not produce a visible pulse. Thus we favor anisotropy, rather than cutoff change as the preferred explanation. This anisotropy could either be temporary or could be a feature that is long lasting but only briefly observed because the asymptotic directions of the detector are changed by the geomagnetic disturbance. We also note that $\mathrm{Kp}$ is only defined in three hour intervals, and is clearly elevated over an interval more extensive than the GRAPES pulse. A more detailed look at the event, using a more fine-grained magnetospheric parameter, is 
clearly required to isolate changes in the cutoff predicted by conventional models from those that might lie outside these models.

\section{Acknowledgements}

We acknowledge the $\mathrm{NMDB}$ database, founded under the European Union's FP7 programme (contract no. 213007) for providing neutron monitor data, and acknowledge individual monitors following the information given on the respective station information sub-page under www.nmdb.eu. Supported in part by US National Science Foundation awards PLR-1245939 and PLR-1341562 and grant RTA5980003 from the Thailand Research Fund.

\section{References}

[1] Evenson, Paul, John Bieber, John Clem and Roger Pyle, South Pole neutron monitor lives again, International Cosmic Ray Conference 32, Beijing, (2011).

[2] Hatton, C. J., The Neutron Monitor, Progress in Elementary Particle and Cosmic Ray Physics $\mathbf{X}$ (1971), American Elsevier Publishing Company, New York.

[3] IceCube Collaboration, M. G. Aartsen et al., The IceCube Neutrino Observatory: Instrumentation and Online Systems, JINST 12 (2017) P03012.

[4] IceCube-Gen2 Collaboration, GeV solar energetic particle observation and search by IceTop from 2011 to 2016, POS (ICRC2017) 132 (these proceedings).

[5] Lin, Zhongmin, John W. Bieber, and Paul Evenson, Electron trajectories in a model magnetosphere: simulation and observation under active conditions, Journal Of Geophysical Research 100 (1995) $23,543-23,549$.

[6] Mangeard, P.-S., D. Ruffolo, A. Sáiz, S. Madlee, and T. Nutaro, Monte Carlo simulation of the neutron monitor yield function, J. Geophys. Res. Space Physics 121 (2016) doi:10.1002/2016JA022638.

[7] Mohanty, P.K., K.P. Arunbabu, T. Aziz et. al., Transient weakening of earth's magnetic shield probed by a cosmic ray burst, PRL 117 (2016) 171101.

[8] Papailiou, M., H. Mavromichalaki, A. Belov, E. Eroshenko and V. Yanke, Precursor effects in different cases of forbush decreases, Solar Phys. 276 (2012) 337-350 DOI 10.1007/s11207-011-9888-1.

[9] Stoker, P.H., Spectra of solar proton ground level events using neutron monitor and neutron moderated detector recordings, International Cosmic Ray Conference 19, La Jolla 4 (1985) 114-117.

[10] Thomsen, M.F., Why Kp is such a good measure of magnetospheric convection, Space Weather 2 (2004) S11004, doi:10.1029/2004SW000089.

[11] Tsyganenko, N.A., Global quantitative models of the geomagnetic field in the cislunar magnetosphere for different disturbance levels, Planet. Space Sci. 35 (1987) 1347-1358. 